\title{
New asymmetric syntheses with boronic esters and fluoroboranes*
}

\author{
Donald S. Matteson ${ }^{\ddagger}$ \\ Department of Chemistry, Washington State University, Pullman, WA 99164-4630, \\ USA
}

\begin{abstract}
Efficient cleavage of sterically hindered boronic esters of asymmetric diols to recover both the diol and a more reactive borane derivative has been a challenging problem. A remarkable borosilicate glass-catalyzed cleavage with thionyl chloride and imidazole proved less useful than hoped for. Potassium bifluoride converts boronic esters to diols and alkyltrifluoroborates. These with chlorosilanes yield alkyldifluoroboranes, which with alkyl azides form secondary amines in high enantiopurity.
\end{abstract}

\section{INTRODUCTION}

Boronic ester chemistry has provided promising routes to asymmetric compounds in high enantiomeric excess. The reaction of (dichloromethyl)lithium with boronic esters of chiral diols (1) followed by zinc chloride-catalyzed rearrangement of the resulting (dichloromethyl)borate complex (2) leads to asymmetric ( $\alpha$-chloroalkyl)boronic esters (3) with diastereoselection often $\sim 100: 1$ at the newly formed stereogenic $\alpha$-carbon, as illustrated in Scheme 1 [1-4]. Reactions of 3 with a wide variety of nucleophiles $\left(\mathrm{Y}^{-}\right)$lead to various asymmetric boronic ester products 4 . Repetition of this process with a second nucleophilic reagent leads to boronic esters (5) containing two adjacent stereogenic centers. If the final $\mathrm{Y}$ group is bound through carbon, the boronic ester intermediate such as $\mathbf{5}$ can undergo peroxidic oxidation to provide an asymmetric secondary alcohol (6). It has been shown that in favorable cases with 1 made from a $C_{2}$-symmetrical diol $\left(\mathrm{R}^{2}=\mathrm{R}^{3}\right)$, sequential double diastereoselection can exceed 1000:1 at each carbon in 6 [5].

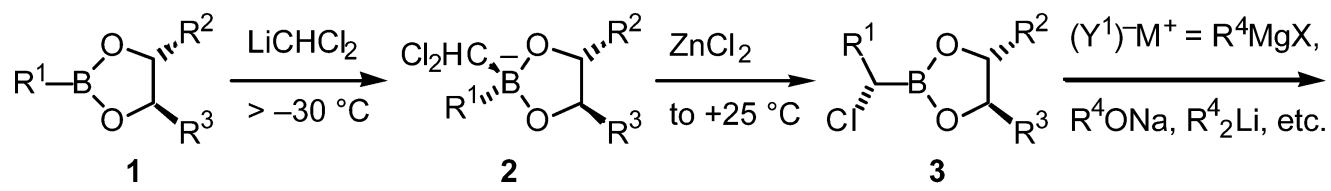

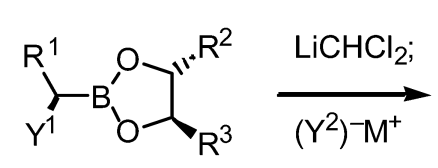

4<smiles>[Y][Y]([R])C([Y])B1OC([R])C([R])O1</smiles>

5

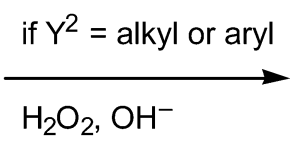

$\mathrm{H}_{2} \mathrm{O}_{2}, \mathrm{OH}^{-}$<smiles>[Y]C([R])C([Y])O</smiles>

6

Scheme 1

\footnotetext{
${ }^{*}$ Lecture presented at the $\mathrm{XI}^{\text {th }}$ International Meeting on Boron Chemistry (IMEBORON XI), Moscow, Russia, 28 July-2 August 2002. Other presentations are published in this issue, pp. 1157-1355.

ॠE-mail: dmatteson@wsu.edu
} 
Useful as this chemistry can be for constructing asymmetric systems that are difficult to assemble by other routes, the low reactivity of boronic esters has proved to be a barrier to some of the useful transformations possible with trialkylboranes [6]. Although conversion of boronic esters to trialkylboranes is merely a matter of repeated alkylation with a Grignard or lithium reagent, and procedures that convert even the highly resistant pinanediol boronic esters to alkylboranes or borinic esters have been developed [7], it would be preferable to convert these sterically hindered boronic esters directly to reactive, highly electrophilic alkylboranes that contain only one carbon-boron bond. Such an approach would circumvent the competitive reactions of different alkyl groups often encountered in trialkylborane chemistry.

\section{CLEAVAGE OF DIOL BORONIC ESTERS TO HALOAMINOBORATES}

This approach appeared to be a promising solution to the efficient cleavage of resistant $C_{2}$-symmetrical diol boronic esters and even pinanediol esters when it was first discovered [8]. The initial observation was that 1,2-dicyclohexyl-1,2-ethanediol (DICHED) benzylboronate $\left(7, \mathrm{R}^{1}=\right.$ benzyl) was cleaved rapidly by thionyl chloride and pyridine in acetonitrile or other aprotic solvents (Scheme 2). Nearly quantitative yields of DICHED sulfite (8) and a pyridine complex of benzylboron chloride, later identified as $\mathbf{9}$, were obtained. The sulfite ester was easily separated by extraction of the acetonitrile phase with pentane, and treatment with base rapidly yielded free DICHED (10). Hydrolysis of the pyridine-benzylboron chloride complex yielded benzylboronic acid $\left(\mathbf{1 1}, \mathrm{R}^{1}=\right.$ benzyl).
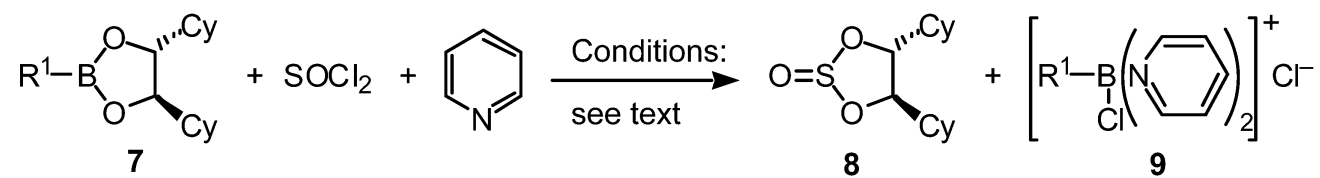

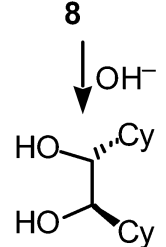

10

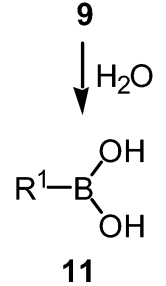

Scheme 2

A number of small-scale test reactions were then run on a variety of compounds, and the sulfite ester production was measured by ${ }^{1} \mathrm{H}-\mathrm{NMR}$. Imidazole provided more rapid and complete cleavage reactions than pyridine. Even pinanediol esters (12) were cleaved (Scheme 3), yielding a diastereomeric pair of pinanediol sulfites (13) and what was later shown to be a complex mixture of monomeric and polymeric imidazole complexes (14), though reactions were slower than with DICHED esters. Sometimes, the reactions did not go to completion in the allotted time, but almost all boronic esters tested yielded some cleavage products.

None of the reactions had been tested on a multigram scale. Strange results soon followed. When a new graduate student (L. Fabry-Asztalos) tried to repeat the work, his initial yields of cleavage prod-

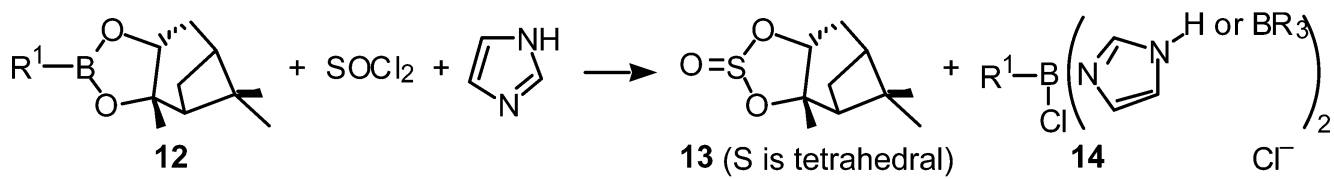

Scheme 3 
ucts were precisely zero! After a number of attempts, he was able to obtain small yields with the most reactive combination, DICHED benzylboronate and imidazole, but the reactions stopped after a few percent completion. We had a bizarre mystery on our hands.

To make a long story short, everything suddenly made sense after Prof. D. C. Dittmer, who had had previous experience with glass surface catalysis [9], suggested that possibility to us. It turned out that the reaction is catalyzed specifically by borosilicate glass, and many DICHED boronic esters and some pinanediol boronic esters can be cleaved efficiently by stirring the reaction mixture with powdered glass. Apparently, the reactions proceed until the glass surface becomes coated with polymeric byproducts. Reactivation of the surface under the reaction conditions proved impossible, and even fresh glass then became inactive before the reaction would proceed much further, but if enough glass surface was available initially the reactions would go in high yield at room temperature within a few minutes. The initial successful small-scale test reactions had been run in flasks that had been etched by repeated washing in alcoholic potassium hydroxide, and the new student used new flasks that only began to have catalytic activity after they had been etched a bit.

The hydrolyses of the alkylboron chloride imidazole complexes were sluggish, and the isolated yields of boronic acids were usually only $\sim 60-70 \%$, though diol recovery was generally nearly quantitative. The conditions are mild and should be generally tolerant of functional groups. However, a further observation on alkylboronic acid chemistry led us to discontinue investigation of the use of this method of boronic ester cleavage in favor of a generally better one.

\section{CONVERSION OF DIOL BORONIC ESTERS TO TRIFLUOROBORATES}

The conditions for conversion of arylboronic acids to aryltrifluoroborates by potassium bifluoride reported by Vedejs and coworkers [10] were tested on one of the alkylboronic acids produced from the chloride-imidazole derivatives and found to work very well. The next obvious step was to attempt the direct cleavage of a DICHED boronic ester (7) as outlined in Scheme 4, and somewhat to our surprise, the reaction proved rapid and efficient at room temperature, directly yielding free DICHED (10) and potassium alkyltrifluoroborate (15) [11].

Even the particularly resistant pinanediol esters (12) are rapidly cleaved to $\sim 70 \%$ free pinanediol (16) and alkyltrifluoroborate (15) at equilibrium under the usual reaction conditions (Scheme 4). The reaction was shown to be reversible by mixing the phenyltrifluoroborate salt $\left(\mathbf{1 5}, \mathrm{R}^{1}=\mathrm{Ph}\right)$ with pinanediol (16) under conditions similar to those used for the usual reactions and showing that $30 \%$ was converted to the pinanediol borate (12) [11].

Several DICHED boronic esters 7 were tested as substrates for the reaction with potassium bifluoride. Reactions were generally run $0.5-2 \mathrm{~h}$ at $\sim 22{ }^{\circ} \mathrm{C}$. For isolation, the methanol was removed under vacuum and the residue was treated with acetonitrile to dissolve the alkyltrifluoroborates (15), DICHED (10), and any unchanged boronic ester 7, leaving the excess unchanged potassium bifluoride

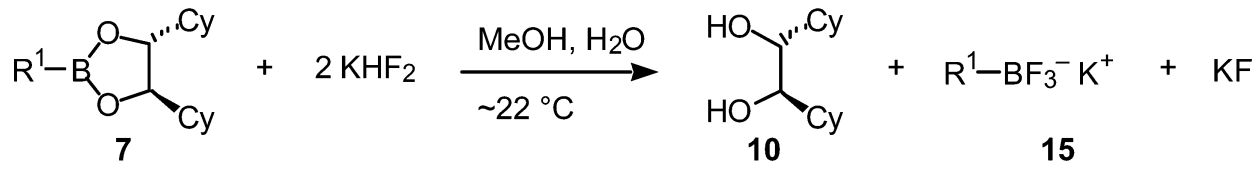

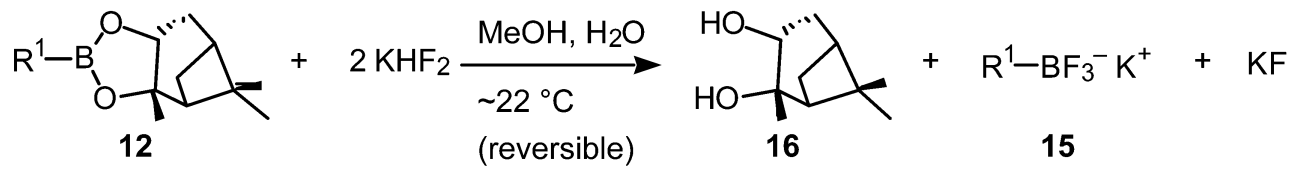

Scheme 4

(C) 2003 IUPAC, Pure and Applied Chemistry 75, 1249-1253 
and product potassium fluoride as an insoluble residue. Pentane extraction removed $\mathbf{7}$ and $\mathbf{1 0}$, though 10 is not very soluble in pentane and can be largely recovered by crystallization from relatively concentrated solutions in acetonitrile/water. Yields of trifluoroborate salts $\mathbf{1 5}$ obtained by concentration of the acetonitrile solutions ranged from $70-91 \%$ for the series $\mathrm{R}^{1}=\mathrm{Ph}$, cyclohexyl, $n$-hexyl, $(S)$ - $\alpha$-chlorobenzyl, $(R)$ - $\alpha$-(benzyloxy)benzyl, $(R)$-4-azido-1-phenylbutyl, and $(S)$-2-octyl. For the same series, DICHED (10) recovery was 86-96\%. Pinanediol esters (12) were not so thoroughly tested, but with $\mathrm{R}^{1}=\mathrm{Ph}$ or $(R)$-4-azido-1-phenylbutyl, conversions were $\sim 70 \%$ [11].

The efficient recovery of the valuable chiral diol is a significant advantage of the potassium bifluoride reaction.

Equilibrium constants of course vary with different substrates, and it appears that even some of the more sterically hindered DICHED boronic esters (7) are not completely converted to alkyltrifluoroborates (15) by potassium bifluoride (work in progress with B. J. Kim). However, it can be difficult to distinguish between incomplete reaction (from a synthetic rather than a theoretical point of view) and reversal during an extraction for workup purposes. We are currently working on this class of reactions to determine all of the parameters that affect the practical results.

\section{ALKYLDIFLUOROBORANE CHEMISTRY}

Vedejs treated the phenyltrifluoroborate salt with trimethylchlorosilane to form phenylboron difluoride, not isolated but used as an intermediate in a further synthesis [10]. This reaction has proved equally applicable to alkyltrifluoroborate salts (15) [11]. However, we have found that silicon tetrachloride reacts faster and achieves rapid conversion to the alkyldifluoroboranes at room temperature.

The chemistry of alkyldifluoroboranes has been largely neglected since the classical syntheses of these compounds were done by McCusker and coworkers in the 1950s [13,14]. Preparation of these extremely air- and moisture-sensitive compounds from alkylboronic anhydrides or alkylboron dichlorides required a fair amount of effort, and there was no obvious advantage in using the difluoroboranes over the more easily prepared chloro analogs. Alkyldichloroboranes have received more attention and continue to produce new chemistry, for example, the interesting aldehyde alkylations recently described by Kabalka and coworkers [15].

The only limitation on the use of inert solvents is that the particular trifluoroborate $\mathbf{1 5}$ have sufficient solubility in the medium to permit reaction. In some cases, plain toluene is useful, others require acetonitrile as a cosolvent. The estimated bond strength for the complex of boron trifluoride with acetonitrile has been calculated to be $\sim 9.1 \mathrm{kcal} / \mathrm{mol}$, not a high barrier to dissociation. Alkylboron difluoride complexes with nitriles will probably be weaker than that, so that even in acetonitrile the alkylboron difluorides should behave as essentially free species.

To demonstrate the synthetic utility of alkyldifluoroboranes, we chose the reaction with azido compounds to form secondary amines. Alkyl azides have long been known to react rapidly with alkylchloroboranes [16,17], but the fluoro analogs had not been tested. Both intermolecular and intramolecular reactions were successful. DICHED $(R)$-2-octylboronate was converted via the difluoroborane and reaction with benzyl azide to $(R)$ - $N$-benzyl- $N$-2-octylamine. Conversion of $(R)$-DICHED or $(S)$-pinanediol $(R)$-4-azido-1-phenylbutylboronate (7 or 12) to the potassium trifluoroborate salt 15 $\left[\mathrm{R}^{1}=(R)\right.$-4-azido-1-phenylbutyl] followed by defluoridation with silicon tetrachloride efficiently yielded $(R)$-2-phenylpyrrolidine (17) after hydrolysis. The overall synthetic route for this process is illustrated in Scheme 5. The synthetic steps utilized are the same as or similar to previously published work [18-20].

The enantiomeric purity of the 2-phenylpyrrolidine produced by this process was expected to be high, and this expectation was verified. Formation of amide derivatives with Mosher's acid chloride [(R)-(-)- $\alpha$-methoxy- $\alpha$-(trifluoromethyl)phenylacetyl chloride] followed by GC/MS analysis indicated enantiomeric purities of $98 \pm 0.4 \%$ for samples of 2-phenylpyrrolidine derived from either the DICHED boronic ester or the pinanediol boronic ester [11]. 

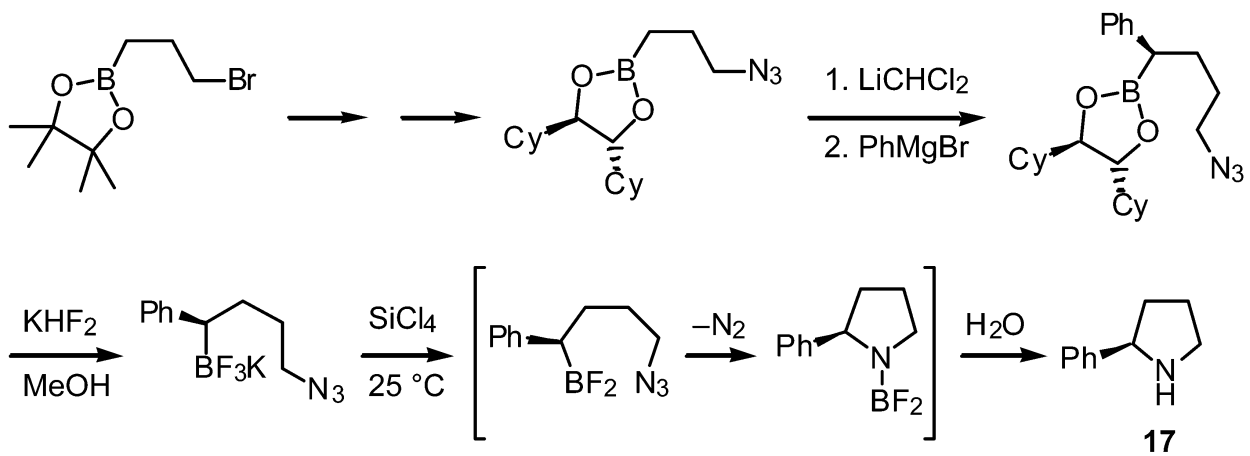

Scheme 5

This preliminary exploration of the chemistry of alkyldifluoroboranes has only demonstrated that at least one process known for the corresponding alkyldichloroboranes can be carried out with the fluorine analogs. It is not expected that all of the chemistry of the fluoro compounds will be closely analogous to what has been observed with alkyldichloroboranes. Fluoride is a much weaker nucleophile toward carbon than chloride, and the alkyldifluoroboranes, being harder Lewis acids than the chloro compounds, may bind more strongly to different nucleophiles. These differences suggest the possibility that alkyldifluoroboranes may show a unique pattern of behavior as alkylborane reagents, and we are actively exploring this chemistry.

\section{ACKNOWLEDGMENT}

We thank the National Science Foundation for support, grant numbers CHE-9613857 and CHE0072788 .

\section{REFERENCES}

1. D. S. Matteson, K. M. Sadhu, M. L. Peterson. J. Am. Chem. Soc. 108, 812 (1986).

2. D. S. Matteson. Chem. Rev. 89, 1535 (1989).

3. D. S. Matteson. Tetrahedron 54, 10555 (1998).

4. D. S. Matteson. J. Organomet. Chem. 581, 51 (1999).

5. P. B. Tripathy and D. S. Matteson. Synthesis 200 (1990).

6. H. C. Brown and B. Singaram. Acc. Chem. Res. 21, 287 (1988).

7. H. C. Brown and M. V. Rangaishenvi. J. Organomet. Chem. 358, 15 (1988).

8. D. S. Matteson, W. C. Hiscox, L. Fabry-Asztalos, G.-Y. Kim, W. F. Siems, III. Organometallics 20, 2920 (2001).

9. D. C. Dittmer and A. F. Marcantonio. J. Org. Chem. 29, 3473 (1964).

10. E. Vedejs, R. W. Chapman, S. C. Fields, S. Lin, M. R. Schrimpf. J. Org. Chem. 60, 3020 (1995).

11. D. S. Matteson and G. Y. Kim. Org. Lett. 4, 2153 (2002).

12. V. Jonas, G. Frenking, M. T. Reetz. J. Am. Chem. Soc. 116, 8741 (1994).

13. P. A. McCusker and L. J. Glunz. J. Am. Chem. Soc. 77, 4253 (1955).

14. P. A. McCusker and H. S. Makowski. J. Am. Chem. Soc. 79, 5185 (1957).

15. G. W. Kabalka, Z. Wu, Y. Ju. Tetrahedron 57, 1663 (2001).

16. H. C. Brown, M. M. Midland, A. B. Levy. J. Am. Chem. Soc. 94, 2114 (1972).

17. H. C. Brown, M. M. Midland, A. B. Levy. J. Am. Chem. Soc. 95, 2394 (1973).

18. D. S. Matteson and R. Soundararajan. Organometallics 14, 4157 (1995).

19. H.-W. Man, W. C. Hiscox, D. S. Matteson. Org. Lett. 1, 379 (1999).

20. R. P. Singh and D. S. Matteson. J. Org. Chem. 65, 6650 (2000). 